\title{
A case report on broad ligament fibroid mistaken as pregnancy
}

\section{Shirish Dulewad, Pooja Chandak*}

Department of Obstetrics and Gynecology, Dr. S. C. G. M. C., Nanded, Maharashtra, India

Received: 25 June 2019

Revised: 17 September 2019

Accepted: 07 October 2019

\section{* Correspondence:}

Dr. Pooja Chandak,

E-mail: drpoojachandak@gmail.com

Copyright: (c) the author(s), publisher and licensee Medip Academy. This is an open-access article distributed under the terms of the Creative Commons Attribution Non-Commercial License, which permits unrestricted non-commercial use, distribution, and reproduction in any medium, provided the original work is properly cited.

\section{ABSTRACT}

Leiomyoma is the commonest benign uterine condition arising from smooth muscles. Extra uterine leiomyoma is rare. The incidence of broad ligament fibroid is less than $1 \%$. Because of its rarity broad ligament fibroid poses specific diagnostic difficulties causing an error in making the final diagnosis and therefore the management. Here we report a case of rare broad ligament fibroid got neglected sadly in this modern era, where the facilities like USG which can easily diagnose this rare entity are readily available. Presented as ANC with no labor pains and accidentally diagnosed as a case of huge broad ligament fibroid. Managed surgically by myomectomy with conservation of uterus and bilateral internal iliac artery ligation for minimizing bleeding.

Keywords: Broad ligament fibroid, Conservative management, Mistaken pregnancy, Need of health care at grass root level, Uterine artery ligation

\section{INTRODUCTION}

Leiomyoma is the most common pelvic tumor of the uterus. Extra uterine leiomyoma may develop in the broad ligament or at other sites where smooth muscle exists. ${ }^{1}$ Among the extra uterine fibroids, broad ligament fibroids are the most common to occur. ${ }^{2}$ Common symptoms of leiomyoma include menstrual disturbances like menorrhagia, polymenorrhoea etc., dysmenorrhea, and symptoms related to pressure caused by the mass like urinary retention or constipation. Incidence of broad ligament fibroid is less than $1 \% .^{3}$ Because of its rarity broad ligament fibroid poses specific diagnostic difficulties causing an error in making the final diagnosis and therefore the management.

Here we present a case of huge broad ligament fibroid mistaken for full term pregnancy by the patient and its management with myomectomy with conservation of uterus and bilateral internal iliac artery ligation.

\section{CASE REPORT}

A 26 years old P1L1 with h/o 9 and half months amenorrhoea with abdominal distension corresponding to full term uterus presented in the ANC OPD considering herself pregnant and not having labor pains despite post EDD. She has not registered for ANC anywhere neither underwent any investigations or USG as her previous delivery was FTND with normal ANC course 4 years back.

On examination we came to know that the patient is not pregnant, she is having a huge abdominal mass arising from pelvis, hard in consistency and non-mobile extending to the xiphisternum for which she underwent USG and CT scan which suggested a huge broad 
ligament fibroid or uterine subserosal fibroid (Figure 1). Tumor markers were done to rule out any malignancy and were normal. Patient was posted for Myomectomy with consent for Hysterectomy if required.

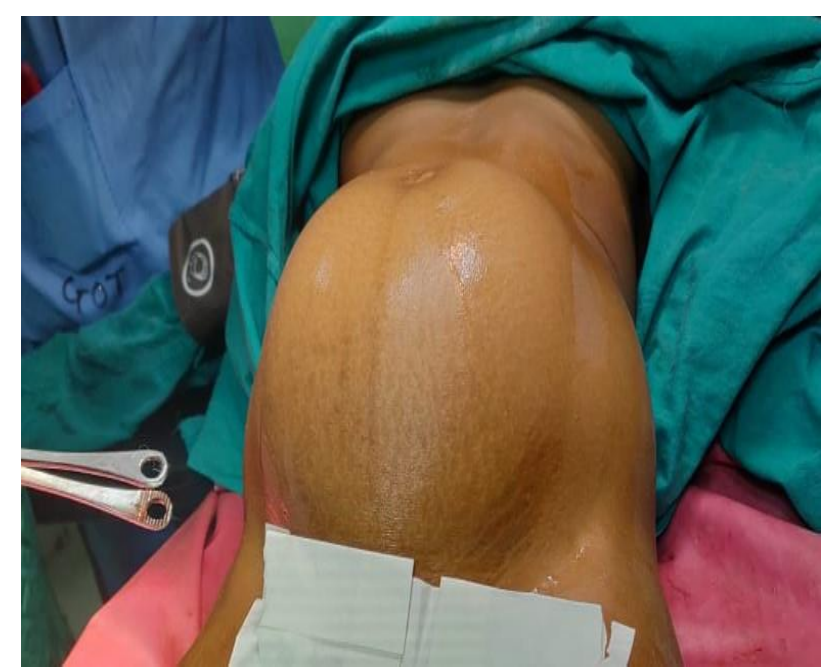

Figure 1: Preoperative image showing globular abdomen simulating full term pregnancy.

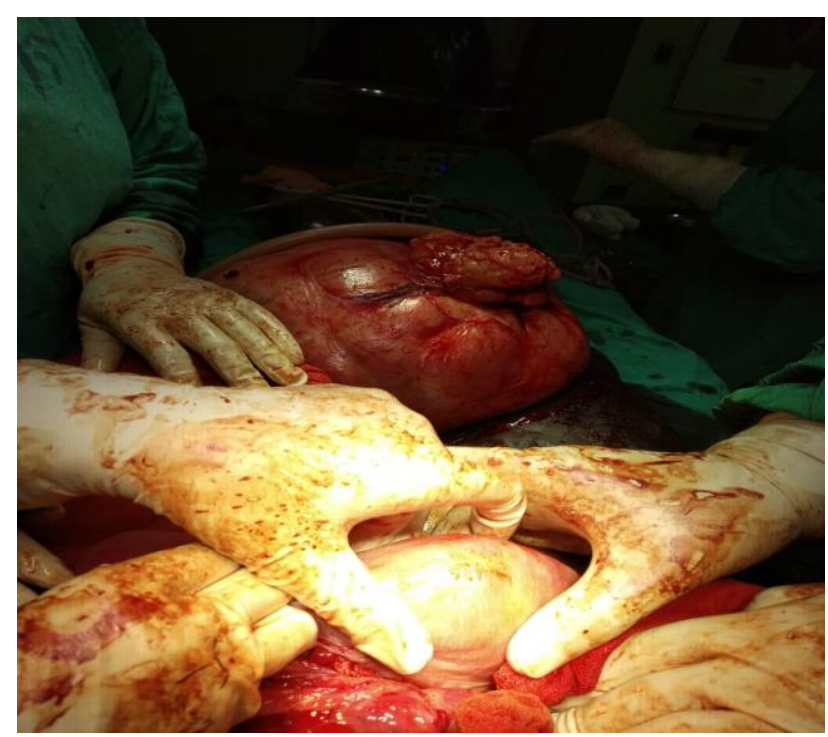

Figure 2: Image taken intraoperatively showing huge fibroid removed with uterus conserved.

Extended pfannenstiels incision was chosen for the procedure. Intraoperative findings were there was a huge left broad ligament fibroid hard in consistency, fixed, and impacted with large dilated many vessels on the surface of the fibroid with ureter running on the surface of fibroid with bulky uterus and bilateral ovaries and fallopian tubes were normal. A huge Broad ligament of the fibroid of around $10 \mathrm{kgs}$ and of $30 * 30 * 30 \mathrm{~cm}$ enucleated taking care not to injure ureter and uterus (Figure 2). Specimen sent for Histopathology reporting. All the precautions were taken to conserve uterus. Bilateral Internal iliac artery ligation was done to minimize the bleeding from myoma bed. Hemostasis achieved and abdomen closed in layers after checking mop and instrument count.

Patient was given 3 blood transfusions intra and postoperatively. Patients postoperative course was uneventful and given discharge on day 12 after removing sutures.

Histopathology report confirmed the diagnosis of fibroid (Leiomyoma).

\section{DISCUSSION}

Fibroids or leiomyoma are the most common benign tumors of the uterus. Rarely, they arise from extrauterine sites the broad ligament is the most common extrauterine site for the occurrence of leiomyoma but with a very low incidence rate of less than $1 \% .^{3}$ Because of its overall low incidence rate, it poses both clinical and radiological difficulty in diagnosis as well as differentiating with an ovarian tumor or uterine subserosal fibroid.

Broad ligament fibroids may be true or false, with $80 \%$ having primary origin in the body of the uterus and $20 \%$ originating within the folds of the broad ligament, known as true broad ligament fibroids. ${ }^{4}$ While in most cases broad ligament fibroids are asymptomatic, ones that achieve large size or undergo degeneration may cause symptoms such as pelvic pain or palpable abdominal mass. ${ }^{5}$ Pain may be present as a result of compression of adjacent organs or torsion of fibroid. Another major concern when dealing with broad ligament fibroids is the complications arising from distortion of pelvic anatomy. ${ }^{6}$ Because of the location and size of broad ligament fibroids, surgery is challenging, especially since surrounding organs such as ureters, intestines, and urinary bladder may be at risk. It is very important that the ureteric course is identified during surgery. ${ }^{7,8}$

\section{CONCLUSION}

Here we report a case of rare broad ligament fibroid got neglected sadly in this modern era, where the facilities like USG which can easily diagnose this rare entity are readily available. Presented as ANC with no labor pains and accidentally diagnosed as a case of huge broad ligament fibroid. Managed surgically by myomectomy with conservation of uterus and bilateral internal iliac artery ligation to minimize the bleeding.

The diagnosis of broad ligament leiomyoma is difficult owing to its rarity, unusual presentation, clinical and radiological features. Thus, it is very important to diagnose it unequivocally. We are reporting this case on account of its rarity and diagnostic difficulties.

In $21^{\text {st }}$ century where medical science is achieving the unimaginable, there are countries like India where women's health gets neglected despite of lot of programs and initiatives undertaken by government as well as 
different health organizations. So, it is necessary that the benefits from these programs should reach the grass root level, then and only then the goal of all these initiatives will be fulfilled in real sense of the term.

Funding: No funding sources

Conflict of interest: None declared

Ethical approval: Not required

\section{REFERENCES}

1. Fasih N, Shanbhogue AK, Macdonald DB, FraserHill MA, Papadatos D, Kielar AZ, et al. Leiomyomas beyond the uterus: unusual locations, rare manifestations. Radiograph. 2008;28:148-93.

2. Hada A, Sen S. Rare case of broad ligament fibroid with cystic degeneration. Int J Reprod Contracept Obstet Gynecol. 2016;5:3226-8.

3. Rajanna DK, Pandey V, Janardhan S, Datti SN. Broad ligament fibroid mimicking as ovarian tumor on ultrasonography and computed tomography scan. J Clin Imaging Sci. 2013;3:8.

4. Rajput DA, Gedam JK. Broad ligament fibroid: a case series. IJSS Case Report Reviews. 2015;1(11):8-11.
5. Bhatla N. Tumours of the corpus uteri. In: Jeffcoats Principles of Gynaecology $6^{\text {th }}$ edn. Arnold Printers, London; 2001:470.

6. Kumar P, Malhotra N. Tumours of the corpus uteri and tumours of the pelvic ligament. In: Jeffcoat's Principles of Gynaecology. $7^{\text {th }}$ ed. New Delhi: Jaypee Brothers Medical Publishers (P) Ltd.; 2008:487-516, 523.

7. Bansal P, Garg D. A case of massive broad ligament leiomyoma imitating an ovarian tumour. J Clin Diagn Res. 2014;8:136-7.

8. Moyle PL, Kataoka MY, Nakai A, Takahata A, Reinhold C, Sala E. Nonovarian cystic lesions of the pelvis. Radiograph. 2010;30:921-38.

Cite this article as: Dulewad S, Chandak P. A case report on broad ligament fibroid mistaken as pregnancy. Int J Reprod Contracept Obstet Gynecol 2019;8:4573-5. 